\title{
Transtornos alimentares e a influência das mídias sociais na autoimagem corporal: um olhar sobre o papel do nutricionista
}

\author{
Eating disorders and the influence of social media on body self-image: a look at the role of the \\ nutritionist \\ Los trastornos alimentarios y la influencia de las redes sociales en la autoimagen corporal: una \\ mirada al papel del nutricionista
}

\section{Resumo}

A autoimagem corporal consiste na representação mental sobre seu próprio corpo e que, quando de difere da idealizada, gera insatisfação. Considerando os padrões de beleza ideal estabelecidos pela mídia, frequentemente indivíduos sofrem de uma insatisfação excessiva, o que pode levar a distorção da autoimagem e transtornos alimentares. Diante disso, o presente estudo buscou realizar um panorama sobre a influência das mídias sociais na autoimagem corporal e na ocorrência de transtornos alimentares, destacando o papel do nutricionista nesses casos. Pode-se observar que as mídias sociais possuem impacto direto na insatisfação corporal, repassando o corpo magro como sinônimo de status, sucesso e sensualidade, com maior destaque para as redes sociais. Nesse cenário, as mais atingidas são as mulheres, principalmente as adolescentes, que passam por mudanças corporais nesse período, ficam mais expostas as mídias e são mais influenciáveis. Casos de transtornos alimentares, como anorexia e bulimia nervosa, podem ser desenvolvidos e constantemente são acompanhados por uma autoimagem distorcida. Por fim, foi possível identificar que o nutricionista, dentro de uma equipe multidisciplinar de tratamento, possui papel fundamental, pois pode atuar tanto no diagnóstico, ao verificar alterações, quanto no tratamento em si, através da reeducação alimentar e outras metodologias, sendo essencial o conhecimento específico do tema.

Palavras-chave: Autoimagem; Transtorno da compulsão alimentar; Mídias sociais.

\begin{abstract}
The body self-image consists of the mental representation of one's own body and, when it differs from the idealized one, it generates dissatisfaction. Considering the ideal beauty standards established by the media, individuals often suffer from excessive dissatisfaction, which can lead to self-image distortion and eating disorders. Therefore, this
\end{abstract}


study sought to provide an overview of the influence of social media on body self-image and the occurrence of eating disorders, highlighting the role of the nutritionist in these cases. It can be observed that social media have a direct impact on body dissatisfaction, conveying the thin body as a synonym for status, success and sensuality, with greater emphasis on social networks. In this scenario, the most affected are women, especially teenagers, who undergo bodily changes during this period, are more exposed to the media and are more influenced. Cases of eating disorders, such as anorexia and bulimia nervosa, can develop and are constantly accompanied by a distorted self-image. Finally, it was possible to identify that the nutritionist, within a multidisciplinary treatment team, has a fundamental role, as he can act both in the diagnosis, by verifying changes, and in the treatment itself, through dietary re-education and other methodologies, being essential the specific knowledge of the topic.

Keywords: Self concept; Binge-eating disorder; Social media.

\section{Resumen}

La autoimagen corporal consiste en la representación mental del propio cuerpo y que, cuando difiere del idealizado, genera insatisfacción. Teniendo en cuenta los estándares de belleza ideales establecidos por los medios de comunicación, las personas a menudo sufren de una insatisfacción excesiva, lo que puede provocar una distorsión de la imagen de sí mismos y trastornos alimentarios. Por lo tanto, este estudio buscó brindar una visión general de la influencia de las redes sociales en la autoimagen corporal y la ocurrencia de trastornos alimentarios, destacando el papel del nutricionista en estos casos. Se puede observar que las redes sociales tienen un impacto directo en la insatisfacción corporal, transmitiendo el cuerpo delgado como sinónimo de estatus, éxito y sensualidad, con mayor énfasis en las redes sociales. En este escenario, las más afectadas son las mujeres, especialmente las adolescentes, que sufren cambios corporales durante este período, están más expuestas a los medios de comunicación y son más influyentes. Los casos de trastornos alimentarios, como la anorexia y la bulimia nerviosa, pueden desarrollarse y van acompañados constantemente de una imagen distorsionada de sí mismo. Finalmente, se pudo identificar que el nutricionista, dentro de un equipo de tratamiento multidisciplinario, tiene un rol fundamental, ya que puede actuar tanto en el diagnóstico, verificando cambios, como en el tratamiento mismo, a través de la reeducación dietética y otras metodologías, siendo imprescindible el conocimiento específico del tema.

Palabras clave: Autoimagen; Trastorno por atracón; Medios de comunicación sociales.

\section{Introdução}

Autoimagem é o termo que designa a imagem que se faz ou que se imagina sobre si mesmo. Trata-se do objeto principal da autoestima, associado ao conhecimento sobre si, suas próprias habilidades e competências, bem como suas percepções, sentimentos, atitudes e ideias (Lima, 2019).

Para Rezende (2019) o conceito da autoimagem corporal abrange a representação que é construída na mente do indivíduo a respeito do seu próprio corpo. A imagem corporal real e a idealizada, muitas das vezes, podem ser completamente diferentes, gerando uma avaliação negativa a respeito de si, ou seja, uma insatisfação com seu corpo, que pode ser causada pela comparação com os padrões ideais de beleza estabelecidos socialmente e internalizados na mente do indivíduo.

Os padrões de beleza sempre estiveram presentes na sociedade, seja através das revistas e dos desfiles de moda, seja pelos programas da televisão. Entretanto, com o aumento do uso da internet, o que se observa é uma maior vulnerabilidade das pessoas a essa influência. A diminuição das relações sociais e a exposição constante a perfis influenciadores está associado à uma autoimagem negativa, baixa autoestima e sentimento de inferioridade (Fernandes, 2019).

Entre a parcela da população mais afetada, encontram-se as meninas adolescentes, que tendem a apresentar uma crescente preocupação com o seu peso e que também fazem parte de uma faixa etária que usa intensamente as mídias sociais. Essas ferramentas digitais têm, em seu sentido problemático, a visualização e upload de fotos como as maiores atividades geradoras de transtornos alimentares (Lira et. al., 2017).

O comportamento alimentar é formado desde a infância e pode ser afetado por diversos pontos como os pais, os amigos e a mídia, além das próprias mudanças pelas quais o corpo passa ao longo dos anos e que pode gerar uma grande insatisfação com a imagem corporal. Em alguns casos, essa insatisfação pode levar a distorções que, consequentemente, podem gerar algum transtorno alimentar. Além destas mudanças, outros fatores podem influenciar a distorção da autoimagem, a saber: bullying; convívio social; percepção distorcida da realidade; ambiente de trabalho; ambiente familiar, insatisfações diversas, entre outros (Bittar; Soares, 2019; Lira et. al., 2017). 
Considerando esse cenário, levantam-se alguns questionamentos, como qual o real nível do impacto que as ferramentas digitais têm causado na autoimagem corporal? Qual relação entre as mídias sociais e os transtornos alimentares atualmente? Qual o papel do nutricionista nesse cenário?

Dessa forma, o presente estudo teve como objetivo descrever a influência das mídias sociais na autoimagem corporal e na ocorrência de transtornos alimentares, com um olhar sobre o papel da nutrição nessa área. Para tanto, teve como objetivos específicos: analisar os conceitos de autoimagem corporal e seus distúrbios; revisar aspectos dos transtornos alimentares; apontar a influência das mídias sociais nesses casos; destacar o papel no nutricionista no tratamento desses transtornos.

\section{Metodologia}

Trata-se de uma revisão narrativa de literatura (RNL), que possui caráter amplo e se propõe a descrever o desenvolvimento de determinado assunto, sob o ponto de vista teórico ou contextual, mediante a análise e interpretação da produção científica existente. Essa síntese de conhecimentos a partir da descrição de temas abrangentes favorece a identificação de lacunas de conhecimento para subsidiar a realização de novas pesquisas. Além disso, sua operacionalização pode se dar de forma sistematizada com rigor metodológico (Brum et al., 2015).

Por meio da busca avançada, utilizando-se os termos delimitadores de pesquisa, Autoimagem and Transtornos da compulsão alimentar and Mídias sociais para levantamento de dados dos últimos 10 anos. Este processo envolveu atividades de busca, identificação, fichamento de estudos, mapeamento e análise.

Os dados coletados para a seleção dos artigos analisados neste estudo atenderam aos seguintes critérios de inclusão: tratar-se de artigo original, ter resumo completo na base de dados no idioma de língua portuguesa, cujo objeto de estudo seja do interesse desta revisão narrativa e que esteja disponível gratuitamente, na íntegra, em formato eletrônico na base de dados, publicado nos últimos dez anos. Já os critérios de exclusão foram: artigos publicados em periódicos não editados no Brasil; artigo de revisão; tese ou dissertação; relato de experiências e que fugissem fora do tema central.

Foram identificadas 418 publicações que foram incluídas de acordo com os critérios adotados neste estudo. Após a leitura dos resultados, verificou-se que somente 57 artigos eram de interesse do objeto de estudo proposto.

\section{Revisão de literatura}

\subsection{Autoimagem corporal}

A autoimagem corporal se constitui como uma representação multifacetada da estrutura corporal e da aparência física, e envolve uma ampla gama de fatores biopsicossociais. É a noção que o indivíduo possui sobre o seu próprio corpo, ilustrando mentalmente o seu tamanho, forma, imagem e os sentimentos relacionados a ele. A construção dessa representação é contínua, com constantes alterações baseadas nas relações da pessoa com o ambiente em que está inserida (Viera et al., 2013).

Aguiar (2014) aponta que a imagem corporal é tanto uma representação mental do que o corpo é na realidade, como também o modo como o corpo é percebido, sendo então fortemente influenciado pelos sentidos, sentimentos e ideias.

Cada sujeito vai ter a sua Imagem Corporal, abrangendo todas as vivências com o seu corpo, refletindo a sua história pessoal, assim como todo o caminho percorrido na construção da sua identidade e as suas relações interpessoais. Em suma, podemos afirmar que a imagem corporal comporta processos psicológicos, cognitivos, emocionais, fisiológicos e sociais, estando todos eles em constantes trocas. É portanto, uma construção multidimensional que descreve amplamente as representações internas da estrutura corporal e da aparência física, em relação a nós mesmos e aos outros (Aguiar, 2014).

Dessa forma, pode-se verificar que a imagem corporal é algo complexo, formado tanto por aspectos internos quanto externos. É importante destacar que quando tratamos de imagem mental também estamos relacionando as imagens sensoriais, percebidas da realidade objetiva pelos sentidos, e as imagens representativas imaginárias, com base na nossa fantasia, que pode 
nos fazer enxergar algo que não é real. Quando se compara essa visão com os padrões que consideramos como socialmente aceitos, podemos gerar a não aceitação com o próprio corpo (Souza \& Reis, 2015).

Os padrões de beleza que construímos e identificamos como objeto de desejo são criados com base na cultura em que estamos inseridos, interiorizando um conjunto de atitudes, crenças, regras, valores e comportamentos que são vivenciados pela sociedade. A partir dessas experiências passamos a nos moldar pelas exigências socialmente aceitas. Considerando que a construção da autoimagem pode ser influenciada por esses fatores, observa-se que a busca excessiva para atingir esse padrão pode desencadear distorções e distúrbios da autoimagem (Loiola, 2014).

\subsection{Transtorno de imagem corporal}

Segundo Lima (2019) o transtorno de imagem corporal é marcado pela distorção ou distúrbio da autoimagem corporal, mesclando a insatisfação corporal com a distorção perceptiva, ou seja, uma percepção do próprio corpo diferente da realidade.

Conforme Almeida et. al., (2018) a distorção da imagem corporal é a discrepância entre o índice de massa corporal (IMC) percebido e o real. Pode levar a ações de controle de peso, ingestão inadequada de nutrientes e distúrbios psíquicos, como transtorno de ansiedade e de humor.

O distúrbio da autoimagem corporal é caracterizado como uma autoavaliação distorcida dos indivíduos sobre a sua autoimagem, fazendo que que se sintam inseguros e imaginando que são vistos com hostilidade e desprezo pelas outras pessoas. Constantemente são associados a transtorno alimentares, como a bulimia, anorexia nervosa e obesidade mórbida, causados por uma alimentação inadequada com falta ou excesso de nutrientes necessários para uma vida saudável (Bracht et. al., 2013).

Embora atinja a todos, se apresenta mais claramente entre as mulheres. Estima-se que nove a cada dez mulheres têm algum nível de insatisfação com a sua aparência. Observa-se também que desde a adolescência as mulheres já apresentam uma autocrítica ao seu estado nutricional, superestimando o peso e se percebendo em sobrepeso ou obesidade (Lima, 2019).

\subsection{Transtornos alimentares}

Transtornos alimentares podem ser definidos como síndromes psicossomáticas em que o paciente é acometido por um medo excessivo de engordar. A partir deles podem surgir diversos efeitos colaterais, como amenorreia, hiperatividade, bradicardia e vômitos, além de transtornos psicológicos, como ansiedade, depressão, fobias, compulsões, preocupação excessiva e atitudes excêntricas com relação à comida (Carvalho \& Ferraz, 2019).

De acordo com Kessler e Poll (2017), essas síndromes alteram o comportamento do indivíduo e possuem uma etiologia multifatorial, associada a elementos genéticos, psicológicos e socioculturais. São fortemente marcados pelo consumo alimentar irregular, com compulsão e obsessão pela comida, dietas restritivas e comportamentos purgativos.

Considerando que desde cedo é passado para as crianças o ideal do corpo magro, os transtornos alimentares podem ser desenvolvidos desde a infância, com alterações na sua alimentação. Também pode ocorrer na adolescência, que é faixa etária com maior prevalência desse tipo de distúrbios, apresentando restrição dietética progressiva e eliminando consumo de alimentos (Barbosa et. al., 2019; Ferreira, 2018).

Nesse grupo estão incluídos o transtorno de pica (ingestão de substâncias não nutritivas), de ruminação (episódios de regurgitação repetida que não podem ser explicados por nenhuma condição médica), transtorno alimentar restritivo/evitativo, anorexia nervosa, bulimia nervosa e o transtorno de compulsão alimentar e ortorexia nervosa. As mais comuns são a anorexia se apresenta com um distúrbio da autoimagem levando o indivíduo a manter dietas com restrição calórica em relação às necessidades nutricionais. Possui uma prevalência de $4 \%$ entre as adolescentes do sexo feminino e pode atingir de 4,9 a $25 \%$ 
da população. A bulimia é marcada por episódios recorrentes de compulsão alimentar, consumindo alimentos além das necessidades nutricionais, seguida de comportamentos compensatórios inapropriados, como o vômito autoinduzido. Possui incidência de 1 a 3\% entre as adolescentes (Menon et al., 2018; Barbosa et. al., 2019)

De acordo com a Sociedade Brasileira de Neuropsicologia (SBNp, 2019), nas últimas décadas, tem-se observado um aumento da incidência da doença, com o dobro do número de casos, atingindo principalmente mulheres, adultas e adolescentes. A proporção é de 10 mulheres para cada homem com algum transtorno alimentar. A anorexia nervosa atinge 8 a cada 100 mil indivíduos por ano e a bulimia, 13 a cada mil indivíduos por ano.

Os transtornos alimentares, em especial a anorexia e a bulimia nervosa, estão associadas a preocupação com a autoimagem corporal, que em geral é insatisfeita ou distorcida. Também são relacionados com a caixa autoestima e a influência das mídias, já que leva em consideração os padrões de beleza culturalmente estabelecidos (Barbosa et. al., 2019).

Por essa razão, a adolescência é considerada um ponto crítico para o desenvolvimento desses transtornos, como um período de mudanças físicas e hormonais, e pelo processo de construção psicológica que constrói a identidade. Ao se comparar com os padrões de beleza, há uma tendência de insatisfação com a autoimagem corporal, que impulsiona a busca por mecanismos para atingir o ideal desejado (Copetti et al., 2018).

Por suas características, esses transtornos afetam o estado nutricional e o metabolismo do indivíduo, estando frequentemente associados a um elevado índice de morbidades. Os casos podem atingir níveis ainda mais graves em um ciclo vicioso, em a pessoa tendo ao isolamento do convívio social pela falta de aceitação e preocupação exagerada com sua alimentação e autoimagem corporal. E à medida que se isola, há o agravamento do seu estado psicológico (Kessler et al., 2017).

\subsection{Influência dos padrões das mídias sociais}

Mídias sociais podem ser entendidas como as recentes tecnologias que possibilitaram novas formas de interação e comunicação, sem fatores limitantes como espaço e tempo. Inicialmente associadas a jornais, revistas, rádio e televisão, hoje se apresentam com maior expressividade através das redes sociais, que são plataformas digitais de comunicação instantânea e em massa (Ribeiro; Mendes, 2019).

Conforme Souza, et. al., (2017), as redes sociais são "aplicativos baseados na internet construídos a partir das funções ideológicas e tecnológicas da Web 2.0". Permitem o compartilhamento de informações, interações entre usuários e troca de conteúdo, chegando a ocupar um papel central na vida das pessoas.

Nos últimos anos, o uso das redes sociais aumentou expressivamente. No ano de 2018, no Brasil, 78\% dos usuários de internet estavam em alguma rede social, sendo alguns exemplos Facebook®, Twitter ${ }^{\circledR}$, Instagram ${ }^{\circledR}$, Snapchat ${ }^{\circledR}$, Whatsapp ${ }^{\circledR}$ e blogs ${ }^{\circledR}$. Esse é um espaço dominado por temas relacionados ao corpo, moda, padrão de beleza, alimentação e atividade física, que se tornam temas impulsionadores de marcas e produtos. Grandes exemplos são as blogueiras ou digital influencers, pessoas com grande número de seguidores e que são utilizadas como agentes de marketing (Costa et. al., 2019).

Além disso, no Brasil, um país com tanta diversidade, a publicidade e a mídia permanecem em uma postura de descaso e desvalorização das características do povo brasileiro, considerando apenas aqueles que atendem a determinado tipo de beleza. Aqueles que não se enquadram nessa padronização, possuem então um corpo "imperfeito" necessitando então de reparos e correções (Almeida, 2017).

Rapidamente, o corpo magro é repassado pela mídia como ideal de status, ascensão social, competência e atratividade sexual. Dessa forma, se você não pertence a esse padrão, passa a ser discriminado, tendo então grande pressão sobre os indivíduos cujo corpos fogem do ideal (Copetti \& Quiroga, 2018). 
Segundo Brito, et.al., (2020), atualmente, o corpo está associado ao consumo, sendo frequentemente utilizado como objeto de um produto do mercado industrial. Considerando que a imagem corporal é construída pelas experimentações dos indivíduos, essa exposição prolongada a essas ideias pode provocar a insatisfação corporal e, consequentemente, as pessoas se veem mais dispostas a investir recursos financeiros e tempo na busca do corpo ideal.

A mídia atua na influência da mente de forma subjetiva, interferindo na autoestima e na percepção de si do indivíduo ao determinar o padrão de beleza. Essas ações geram resultados físicos e psicológicos, sendo os principais os transtornos alimentares, como bulimia e anorexia, práticas intensas de atividades físicas, distorção da autoimagem corporal, baixa autoestima e, por fim, pode levar ao óbito (Castro et. al., 2019).

Estudos demonstram que a exposição prolongada a imagens de pessoas que são tidas como padrão de beleza está associada a insatisfação corporal, conforme Lima (2019). Os resultados apontam que ao serem expostas a essas imagens houve uma redução na satisfação com o próprio corpo, tanto entre homens quanto as mulheres. Aqueles que já possuíam algum tipo de insatisfação, apresentaram um aumento na intensidade dela, principalmente as mulheres.

Novamente, as mulheres são as mais atingidas por essa insatisfação. Os homens aparecem com $85,7 \%$ e as mulheres com 55,1\%. Já em relação à insatisfação para os homens, de modo leve, moderado e extremo, respectivamente, aparecem com $7,6 \%, 4,2 \%$ e $2,5 \%$. E para as mulheres desses mesmos modos, respectivamente, aparecem com $24,6 \%, 8,5 \%$ e $11,9 \%$. (...) a mídia causa insatisfação corporal, delimitando, orientando e impondo de modo que leva as mulheres a terem o desejo de modificarem seus corpos de algum modo, e aquelas que não se adequam ao padrão socialmente imposto que é magro e definido, podem passar a se verem de forma negativa (Castro et. al., 2019).

Como as adolescentes possuem maior facilidade de acessar as redes sociais, são as consumidoras assíduas desse tipo de conteúdo e acabam sendo as mais atingidas. Atualmente, temos uma geração que nasceu no meio digital e que de tanto ser induzidas aos corpos de padrões estéticos específicos, acaba acreditando que isso é o correto e é a partir daí que surge a insatisfação corporal. A busca por esse padrão pode levar a dietas, procedimentos invasivos e rotina de exercícios, porém ao continuar sendo exposta a mídia, pode voltar a ter insatisfações e desejar novas transformações corporais, algo que pode culminar em distúrbios de autoimagem corporal e alimentares (Barros, 2019).

De acordo com Silva (2020), esse é um fator preocupante, já que as adolescentes representam um grupo sensíveis a essas influências. As mudanças biológicas e emocionais enfrentadas nesse período são difíceis de lidar, deixando-os mais vulneráveis e com um sentimento de rejeição perante o seu corpo, que não segue aos padrões estabelecidos como belos e perfeitos.

Vargas (2014) aponta que inicialmente o conflito entre a imagem corporal e o desejo pelo corpo perfeito não se caracteriza como um problema, porém isso pode se agravar quando determinada característica se torne um padrão a ser alcançado. A partir daí se abre um leque de possibilidades comportamentais, como transtornos alimentares, jejum prolongado, dietas rigorosas, indução de vômitos e adoção de práticas prejudiciais à saúde, como exercícios físicos exacerbados e cirurgias plásticas.

Acirrados problemas comportamentais, envolvendo hábitos alimentares, podem repercutir negativamente na percepção da imagem corporal, especialmente entre jovens. De um polo a outro, da obesidade à anorexia, jovens desenvolvem distúrbios de conduta alimentar comprometendo sua saúde e qualidade de vida, sempre tendo como espelho as pessoas expostas nas redes sociais, que aparentam e desfilam com o aval de uma suposta perfeição (Silva, 2020).

Através das mídias sociais, indivíduos estimulam suas ideias de apoio aos transtornos alimentares de forma anônima, em grupos chamados "Pró-Ana" (prol da anorexia) e "Pró-Mia" (prol da bulimia). Através dessas páginas, eles incentivam as práticas anoréxicas e bulímicas, defendendo um estilo de vida que tem a restrição alimentar como meio de alcançar o padrão 
corporal de magreza extrema. Dessa forma, são repassadas posturas extremamente prejudiciais a saúde para um grupo cada vez maior de usuários (Rodrigues, 2019).

Segundo Brito et.al., (2020), com a pandemia de Covid-19 e o uso excessivo das redes sociais, houve o aumento do descontrole alimentar, depressão, distúrbios do sono, entre outros. O quadro ansioso aumenta o receio de engordar e perder músculos e, em grande parte dos casos, tem início por pressões sociais repassadas na mídia.

\subsection{Nutrição nos casos de transtornos alimentares e de autoimagem corporal}

Conforme vimos, a insatisfação corporal acentuada pode levar a transtornos de imagem corporal e, consequentemente, a transtornos alimentares. Sant'anna (2014) destaca a série de complicações relacionadas a desnutrição que podem acometer indivíduos com transtornos alimentares. A anorexia nervosa pode levar ao comprometimento cardiovascular desidratação, distúrbios eletrolíticos, distúrbios na motilidade gastrointestinal, infertilidade e hipotermia. Já a bulimia, por manter o peso corporal próximo ao normal ou um leve sobrepeso, pode esconder a doença por longos períodos, por não levar a um estado nutricional tão devastado como ocorre com a anorexia.

Um tratamento eficiente de qualquer transtorno alimentar deve ser composto por uma equipe multidisciplinar, composta por médicos, nutricionistas, psiquiatras, educadores físicos, entre outros. O objetivo é conduzir o indivíduo a estabilização nutricional para o padrão saudável, com conscientização do comportamento inadequado e até o uso de medicamentos para controle de impulsos (Oliveira, 2019).

Nesse sentido, é importante destacar a função do nutricionista, que é seu exercício profissional deve identificar e embasar um padrão alimentar que garanta uma vida plena e saudável. Através do seu estudo sobre a composição dos alimentos e as necessidades nutricionais de cada indivíduo, atua em estados de saúde e doença distintos (Lima, 2019).

O nutricionista é capaz de realizar diagnóstico sobre transtornos alimentares e distúrbios de autoimagem, ao identificar variações do apetite e perturbações da imagem corporal, alertando o profissional sobre a possibilidade desses distúrbios. A partir daí, deverá desenvolver, em conjunto com uma equipe, ações busquem devolver ao indivíduo um peso saudável e um relacionamento sadio com o alimento. Isso pode ser obtido através do desenvolvimento das seguintes atitudes no paciente: respeito em relação à fome físico, bem como a vontade de comer; aceitação das oscilações na alimentação dependendo do humor e da situação social; e sensação de prazer por meio da alimentação (Leite et al., 2020).

Ao atuar na equipe de tratamento, cabe ao nutricionista a orientação e educação nutricional. Farias e Rosa (2020) abordam como deve ser as condutas nesse processo: As condutas nutricionais utilizadas no tratamento de transtornos alimentares almejam reverter alterações funcionais, recuperar o estado nutricional, promover um padrão alimentar que atenda às necessidades e as recomendações nutricionais, sugerir mudanças no comportamento alimentar, quando inadequadas e, por fim, melhorar a relação paciente alimento (Farias \& Rosa, 2020).

$\mathrm{O}$ tratamento nutricional de pacientes com transtornos alimentares envolve duas fases distintas: o educacional e o experimental. A fase educacional é a primeira a ser realizado e pode ser feita por qualquer profissional graduado. Inclui a coleta e transmissão de informações, oferecendo uma correta educação alimentar para o paciente e sua família. A fase experimental possui objetivos mais terapêuticos e deve ser realizada por profissional treinado, com conhecimentos básicos de psicologia. Consiste em ajudar o paciente a separar comportamentos relacionados com o peso e o alimento de sentimentos e questões psicológicas, através da reeducação alimentar e aconselhamentos acerca do comportamento alimentar (Kachani, 2012).

O núcleo familiar deve ser envolvido no tratamento como fora de alterar a cultura alimentar desenvolvida por ele. O grande desafio é desconstruir possíveis aspectos relacionados aos distúrbios alimentares impostos e o compartilhamento de experiências com o decorrer do tratamento. Uma modalidade frequentemente aplicada é a inclusão psicoeducativa, que 
trabalha a terapia em grupo familiar com observações das necessidades individuais e exploração dos sentimentos emocionais, angústia e ansiedade. Tal modalidade tem apresentado resultados positivos na recuperação do doente (Sant’anna, 2014).

Sant'anna (2014) também aponta que o tratamento nutricional vem acompanhado da aferição de medidas antropométricas do paciente, como peso, altura dobras e circunferências, além de buscar compreender as restrições alimentares, a relação com o alimento e as crenças errôneas. O conhecimento de psicologia, psiquiatria e técnicas de terapia é fundamental.

Conforme Kachani (2012), na prática clínica da nutrição de pacientes com transtornos alimentares pode ser dificultada. A aferição de medidas antropométricas, como a pesagem, pode se transformar em um momento dramático, prejudicando a consulta, principalmente se o profissional não está capacitado para isso.

Nutricionistas que atuam no tratamento dos TAs [Transtornos Alimentares] enfrentam uma complexa dinâmica humana, que pode se tornar especialmente estressante em razão da importância das questões alimentares da doença. Portanto, precisam desenvolver algumas habilidades psicoterápicas - sempre apoiados pelos outros profissionais da equipe - para compreensão do potencial de comportamentos disfuncionais, a fim de que não concordem inconscientemente com o estilo de vida dos pacientes (Kachani, 2012).

A literatura dispõe diversas ferramentas para os nutricionistas atuarem nessa área, sendo as mais utilizadas a Educação Alimentar e Nutricional e a Técnica Cognitivo-comportamental, por apresentarem resultados eficazes. Porém, é necessário que o profissional possua uma boa base teórica sobre elas (Farias \& Rosa, 2020).

Portanto, é importante ter um bom conhecimento específico sobre o tema para tratar o paciente e desenvolver a melhor abordagem. Porém, também é preciso ter em mente que nenhuma modalidade deve ser aplicada de maneira isolada, mas de maneira integrada com o tratamento desenvolvido por toda a equipe multidisciplinar, que deve funcionar como uma rede se sustentação para o paciente (Oliveira, 2019).

\section{Conclusão}

A insatisfação corporal pode chegar a estados agravantes, gerando transtornos e distúrbios. A autoimagem corporal transtornada é um deles, caracterizada como uma visão descontente com o próprio corpo, muitas vezes longe da realidade e que frequentemente está associada a transtornos alimentares, onde o indivíduo partindo de um medo excessivo de engordar, sofre alterações na sua relação com o alimento.

Considerando que a autoimagem corporal é um aspecto construído ao longo da vida, a exposição frequente a mídias sociais, que em geral estabelecem um padrão de corpo ideal, é um fator preocupante. Tido como sinal de status e sucesso, o corpo magro ainda é amplamente divulgado, é um estilo de vida que buscam, assim ele é repassado como tendência pelas blogueiras e influencers. Foi possível observar que esses fatores propiciam a distorção da imagem corporal e ao desenvolvimento de transtornos alimentares, atingindo principalmente as mulheres.

Com o grande aumento do uso das tecnologias e o maior tempo de exposição a elas, cada vez mais essa se torna uma situação relevante, sendo de extrema importância a capacitação dos nutricionistas para atuarem no tratamento desses casos. Em uma equipe multidisciplinar, eles são capazes de diagnosticar e tratar esses casos, com reeducação alimentar e outros métodos de terapia. Para tanto, destaca-se a grande necessidade de conhecimento específicos sobre o tema.

Por fim, estudos futuros devem ser idealizados para que novas pesquisas aprofundem esse impacto para melhor esclarecimento das consequências nesses distúrbios de autoimagem e transtornos alimentares. Além disso, pesquisas futuras tratando sobre o uso sensato e adequado das mídias sociais poderiam ajudar a proporcionar uma autonomia maior aos jovens para o uso criterioso do conteúdo consumido e menor impacto na forma em que se alimentam e enxergam suas mudanças corporais. 


\section{Referências}

Aguiar, S. F. A. (2014). O Meu Corpo e Eu: a imagem corporal e a autoestima na adolescência. Dissertação (Doutorado pelo Curso de Psicologia Clínica). Instituto Universitário Ciências Psicológicas, Sociais e da Vida, Lisboa, Portugal. https://repositorio.ispa.pt/handle/10400.12/5028.

Almeida, A. P. (2017). A Influência da Mídia na Distorção da Imagem Corporal de Adolescentes. TCC (Trabalho de Conclusão do Curso de Educação Física), Centro Universitário de Brasília, Brasília. https://repositorio.uniceub.br/jspui/bitstream/prefix/13105/1/21451004.pd.

Almeida, S. G., Almeida, A. G., Santos, A. L. \& Silva, M. L. (2018). A Influência de uma Rede Social nos Padrões de Alimentação de Usuários e Profissionais de Saúde Seguidores de Perfis Fitness. Ensaios e Ciência: C. Biológicas, Agrárias e da Saúde. 22(3), p. 194. Editora e Distribuidora Educacional. https://revista.pgsskroton.com/index.php/ensaioeciencia/article/view/4396.

Barbosa, A. L. P., Enetério, N. G. P., Rolindo, J. M. R. \& Miranda, H. S. L. (2019). Psicologia e Transtornos Alimentares: produção científica sobre anorexia $e$ bulimia nervosa. produção científica sobre Anorexia e Bulimia Nervosa. Repositório Institucional. http://repositorio.aee.edu.br/jspui/bitstream/aee/1061/1/Psicologia\%20e\%20Transtornos\%20Alimentares-

Produção\%20Científica\%20sobre\%20Anorexia\%20e\%20Bulimia\%20Nervosa.pdf.

Barros, Y. I. B. (2019). Imagem Corporal e a Influência da Mídia na Construção do Corpo Feminino. TCC (Trabalho de Conclusão do Curso de Enfermagem na Universidade Católica de Salvador), Salvador. http://ri.ucsal.br:8080/jspui/bitstream/prefix/945/1/TCCYURIBARROS.pdf.

Bittar, C. \& Soares, A. (2020). Mídia e comportamento alimentar na adolescência. Cadernos Brasileiros de Terapia Ocupacional. 28(1), 291-308. https://www.scielo.br/j/cadbto/a/mfTpzZ6F3YhywBGx5tVLkgx/?format=pdf\&lang=pt.

Bracht, C. M., Piasetzki, C. T. R., Busnello, M. B., Berlezi, E. M., Franz, L. B. B. \& Boff, E. T. O. (2013). Percepção da Autoimagem corporal, estado nutricional e prática de atividade física de universitários do Rio Grande do Sul. $O$ Mundo da Saúde. São Paulo, 37(3), 343-353. https://pesquisa.bvsalud.org/portal/resource/pt/mis-36808.

Brito, A. A., Thimóteo, T. B. \& Brum, F. (2020). Redes Sociais, suas Implicações sobre a Imagem Corporal de Estudantes Adolescentes e o Contexto da Pandemia do Coronavírus (COVID-19). Revista Temas em Educação Física Escolar. 5(2), 105-125. http://cp2.g12.br/ojs/index.php/temasemedfisicaescolar/article/download/3032/1882.

Brum, C. N. de., Zuge, S. S., Rangel, R. F., Freitas, H. M. B de., Pieszak, G. M. Revisão narrativa da literatura: aspectos conceituais e metodológicos na construção do conhecimento da enfermagem. In: Lacerda, M. R.; Costenaro, R. G. S. Metodologia de pesquisa para a enfermagem a saúde da teoria a prática. $1^{\mathrm{a}}$ ed. Porto Alegre: Moriá, 2015. p. 123-142.

Carvalho, M. A. \& Ferraz, N. G. (2019). A Relação entre a Autoimagem e o Transtorno Alimentar em Atletas de Ginástica Rítmica de 12 a 13 Anos. TCC (Trabalho de Conclusão de Curso de Educação Física), Universidade de Brasília, Brasília. https://bdm.unb.br/handle/10483/26674.

Castro, A. P., Lopes, N. E., Moreira, T. B. V., Oliveira, T. A. \& Rodrigues, C. M. M. (2019). Os Impactos da Mídia na Imagem Corporal. 2019. https://www.repositoriodigital.univag.com.br/index.php/Psico/article/download/605/602.

Copetti, A. V. S. \& Quiroga, C. V. A influência da mídia nos transtornos alimentares e na autoimagem em adolescentes. Revista de Psicologia da Imed. 10(2), 161-177. https://seer.imed.edu.br/index.php/revistapsico/article/view/2664.

Costa, M. L., Araújo, D. F. S., Cassiano, M. H., Figueirêdo, H. A. O., Oliveira, V. T. L., Barbosa, I. R. B. \& Silva, F. H. V. C (2019). Associação entre o uso de mídias sociais e comportamento alimentar, percepção e checagem corporal. Brazilian Journal of Health Review. 2(6), 5898-5914. https://www.brazilianjournals.com/index.php/BJHR/article/download/5399/4928.

Farias, C. T. S. \& Rosa, R. H. A educação alimentar e nutricional como estratégia no tratamento dos transtornos alimentares. Brazilian Journal of Health Review. 3(4), p. 10611-10620. https://www.brazilianjournals.com/index.php/BJHR/article/viewFile/15623/12853.

Fernandes, K. (2019). Impacto das Mídias Sociais sobre a Insatisfação Corporal e Risco de Transtornos Alimentares e Depressão em Estudantes da Universidade Federal de Ouro Preto. TCC (Trabalho de Conclusão do Curso de Nutrição), Universidade Federal de Ouro Preto, Ouro Preto. https://www.monografias.ufop.br/handle/35400000/2527.

Ferreira, T. D. (2018). Transtornos Alimentares: principais sintomas e características psíquicas. Revista Uningá, Maringá. 55(2), 169-176. http://revista.uninga.br/index.php/uninga/article/view/176.

Kachani, A. T. (2012). Checagem do Corpo em Transtornos Alimentares: relação entre comportamentos e cognições. Tese (Doutorado) - Curso de Ciências, Faculdade de Medicina, Universidade de São Paulo, São Paulo. https://www.teses.usp.br/teses/disponiveis/5/5160/tde-25052012162049/publico/AdrianaTrejgerKachani.pdf.

Kessler, A. L. \& Poll, F. A. (2018). Relação entre imagem corporal, atitudes para transtornos alimentares e estado nutricional em universitárias da área da saúde. Jornal Brasileiro de Psiquiatria. 67(2), 118-125. https://www.scielo.br/j/jbpsiq/a/nyLgzvS6nXQQPTFdqbGzg3w/abstract/?lang=pt.

Leite, R. P. P., Diniz, T. M. \& Aoyama, E. A. O Papel da Nutrição Comportamental no Tratamento dos Transtornos Alimentares e na Distorção da Imagem. Rebis: revista brasileira interdisciplinar de saúde. 2(4), 65-69. https://revistarebis.rebis.com.br/index.php/rebis/article/view/159.

Lima, R. A. R. (2019). Relação entre Mídias Sociais e Transtornos de Autoimagem em Mulheres. TCC (Trabalho de Conclusão do Curso de Nutrição) Centro Universitário de Brasília, Brasília. https://repositorio.uniceub.br/jspui/bitstream/prefix/13488/1/21604814.pdf.

Lira, A. G., Ganen, A. P. \& Lodi, A. S., Alvarenga, M. S. (2017). Uso de redes sociais, influência da mídia e insatisfação com a imagem corporal de adolescentes brasileiras. Jornal Brasileiro de Psiquiatria. 66(3), 164-171. https://www.scielo.br/j/jbpsiq/a/6NrPypcRchnc3 5RH9GLSYwK/?lang=pt\&format=pdf. 
Research, Society and Development, v. 10, n. 16, e191101623277, 2021

(CC BY 4.0) | ISSN 2525-3409 | DOI: http://dx.doi.org/10.33448/rsd-v10i16.23277

Loiola, R. F. (2014). Análise Discursiva da Autoimagem Corporal de Mulheres em Diferentes Idades. Tese (Doutorado) - Curso de Estudos Linguísticos, Universidade Federal de Minas Gerais, Belo Horizonte. https://repositorio.ufmg.br/handle/1843/MGSS-9R3LGP.

Menon, A. M., Blanco, M. B. \& Bernardelli, M. S. (2019). Ações de Intervenção e Orientação Nutricional para Estudantes com Transtornos Alimentares no Brasil: uma revisão sistemática de literatura. Revista Conhecimento Online, Novo Hamburgo. $2(11)$, 93-113. https://periodicos.feevale.br/seer/index.php/revistaconhecimentoonline/article/view/1570.

Oliveira, T. C. (2019). A Relação entre a Autoimagem Corporal e o Risco de Transtornos Alimentares em Estudantes do Curso de Nutrição em Cuiabá-MT. TCC (Trabalho de Conclusão do Curso de Nutrição) - Universidade Federal de Mato Grosso, Cuiabá. https://bdm.ufmt.br/bitstream/1/1627/1/TCC_2019_Tatiane\%20Cortezi\%20Oliveira.pdf.

Rezende, B. B. (2019). Transtornos Alimentares: a influência das mídias sociais na percepção da imagem corporal de jovens e adolescentes. TCC (Trabalho de Conclusão do Curso de Nutrição) - Centro Universitário de Brasília, Brasília. https://repositorio.uniceub.br/jspui/bitstream/prefix/13469/1/21600372.pdf.

Ribeiro, T. S. \& Mendes, A. C. S. (2019). Influência das Mídias Sociais na Insatisfação da Imagem Corporal dos Acadêmicos de Educação Física de Tubarão. 2019. https://riuni.unisul.br/bitstream/handle/12345/10024/ARTIGO-tcc.pdf?sequence=1\&isAllowed=y.

Rodrigues, D. S. S. (2019). A Influência das Mídias Sociais na Autoimagem da Mulher. 2019. TCC (Trabalho de Conclusão do Curso de Psicologia) -
Universidade
do Extremo
Sul
Catarinense,
Criciúma. http://repositorio.unesc.net/bitstream/1/7153/1/DANIELA\%20DA\%20SILVA\%20DE\%20SOUZA\%20RODRIGUES.pdf.

Sant'anna, A. C. N. (2014). Transtorno Alimentar na Adolescência. 2014. TCC (Trabalho de Conclusão do Curso de Nutrição) - Faculdade Católica Salesiana do Espírito Santo, Vitória. https://www.ucv.edu.br/fotos/files/TCC_ANDRESSA_NUTRICAO.pdf.

Silva, P. H. G. (2020). A Influência da Mídia na Autoimagem de Adolescentes: uma análise do discurso nas redes sociais. Revista Philologus. 26(76), 79-89. Disponível em: http://www.filologia.org.br/rph/ANO26/76supl/06.pdf.

Sociedade Brasileira de Neuropsicologia. (2019). Boletim SBNp. 2(10). https://sbnpbrasil.com.br/wp-content/uploads/2019/11/24-Boletim_Out-2019.pdf.

Souza, G., Freitas, T. G. \& Biagi, C. R. A Relação das Mídias Sociais na Construção da Autoimagem na Contemporaneidade. Akrópolis: Revista de Ciências Humanas da UNIPAR. 25(2), 117-128. Universidade Paranaense. https://revistas.unipar.br/index.php/akropolis/article/download/6426/3685.

Souza, G. M. \& Reis, L. F. R. (2015). A Percepção da Autoimagem Corporal de Estudantes Universitários do Primeiro Ano do Curso de Psicologia do Centro Universitário Católico Salesiano Auxilium de Lins numa Perspectiva Psicodramática. TCC (Trabalho de Conclusão do Curso de Psicologia) - Centro Universitário Católico Salesiano Auxilium, Lins. http://www.unisalesiano.edu.br/biblioteca/monografias/58539.pd.

Vargas, E. G. A. (2014). A Influência da Mídia na Construção da Imagem Corporal. Revista Bras. Nutri. Clin. 29(1), 73-75. http://www.braspen.com.br/home/wp-content/uploads/2016/12/12-A-influencia-da-midia-na-construcao-da-imagem.pdf.

Vieira, T. M. R. A., Loiola, R. F., \& Alves, L. M. (2013). Autoimagem Corporal: uma revisão sistemática nas diferentes áreas da saúde. Revista Tecer, Belo Horizonte. 6(11), 166-177. https://www.metodista.br/revistas/revistas-izabela/index.php/tec/article/view/385. 